\title{
TRAVEL HISTORIES AS RISK FACTORS IN THE ANALYSIS OF URBAN MALARIA IN COLOMBIA
}

\author{
LYDA OSORIO, JIM TODD, AND DAVID J. BRADLEY \\ International Center for Medical Research and Training, Cali, Colombia; Department of Infectious and Tropical Diseases, London \\ School of Hygiene and Tropical Medicine, London, United Kingdom
}

\begin{abstract}
Self-reported travel histories were used in a case-control study to determine whether movement of local residents to neighboring endemic areas was a risk factor for malaria in the town of Quibdo, Colombia. Multivariate analyses showed that among residents of Quibdo, traveling to an endemic area 8-14 days before disease onset was the strongest risk factor for both Plasmodium falciparum (adjusted odds ratio [OR] $=28.96,95 \%$ confidence interval $[\mathrm{CI}]=13.9-60.32$ ) and $P$. vivax (adjusted $\mathrm{OR}=14.24,95 \% \mathrm{CI}=5.27-38.46$ ) malaria. For $P$. falciparum, individuals who did not travel outside Quibdo during the 8-14 days before disease onset, but who reported traveling 1-7, 15-21, or 22-30 days before disease onset also had an increased risk of malaria. Conversely, use of protection against mosquitoes was negatively associated with $P$. falciparum. These results highlight the need for malaria control measures that target mobile populations. A definition of imported malaria that allows distinction of imported from autochthonous cases in Quibdo town is proposed.
\end{abstract}

\section{INTRODUCTION}

Population movement has long been recognized to contribute to the epidemiology of malaria. ${ }^{1}$ It is particularly relevant to the epidemiology of urban malaria since, on one hand, urban centers usually offer employment and better living conditions, and therefore experience a high rate of immigration and circular migration from both endemic and non-endemic areas. On the other hand, malaria transmission in urban areas is generally lower than in rural areas, ${ }^{2}$ and an increased risk of malaria has been observed among urban travelers on visiting rural areas ${ }^{3-5}$ compared with those who did not travel. Nevertheless, chemoprophylaxis, or other individual protection measures are not systematically recommended in these circumstances, unlike the situation for short-term travelers from non-malarious countries to endemic countries. ${ }^{6}$

Whether mobile populations should be targeted for malaria control measures is relevant to Colombia because population movement due to conflict and economic necessity may be one of the factors that have contributed to the disease burden. Urban transmission of malaria has been reported for many years in the Pacific Coast region, Department of Antioquia, and the Amazon-Orinoco River basins of Colombia. More recently, malaria has been reintroduced into urban areas in the central region, and on the Atlantic coast. Reintroduction of malaria into these municipalities was associated with the arrival of populations displaced from rural areas to peri-urban areas, and subsequent transmission to local residents. ${ }^{7}$

Administratively, Colombia is divided into a capital district (Bogota) and 32 departments. Each department is divided into municipalities and these are subdivided into villages. The department of Choco is one of the most malarious areas, with annual parasite incidences 4-5 times the national average. Choco is located in northwestern Colombia, along the Pacific coast. The conditions for malaria transmission exist in all the municipalities in the department, with annual parasite incidences ranging from 1 to 90.5 microscopically confirmed cases/1,000 inhabitants in 1999. Malaria is mainly rural, but urban transmission is reported in eight towns in the department, among which Quibdo, the departmental capital, has the highest population (95,509 in 2002) (Choco Vector Borne Diseases Control Program, unpublished data). In the town of Quibdo, a total of 902 and 2,417 cases were diagnosed in 2000 and 2001, respectively (Choco Department of Health, unpublished data). These numbers include cases diagnosed in residents of rural areas and of other municipalities who may come to town in search of diagnosis and treatment, as well as local residents. Thus, malaria reported in Quibdo comprises both imported and autochthonous cases.

The magnitude of imported malaria and its contribution to the local malaria situation in Quibdo is unknown. Currently, malaria cases diagnosed in Quibdo are not classified according to the likely origin of infection. This prevents the malaria control program from accurately quantifying the incidence of malaria in the urban area, and identifying the neighborhoods that should be targeted for control measures.

In the present study, we investigated the risk factors for malaria in residents of Quibdo. We were particularly interested in the risk of traveling to endemic areas at different time periods, and the risk of malaria in local residents who do not travel in comparison with that of residents of areas outside the town. This information proved useful in highlighting the need for malaria control measures that target mobile populations. A definition of imported malaria that allows distinction of imported from autochthonous cases in Quibdo town is proposed.

\section{METHODS}

Study area. The town of Quibdo serves as a river port and commercial center of the region, and many people circulate between the rural and the urban areas. People from the rural areas come to the town in search of health services, education, and temporary jobs, while people from the urban area go to the countryside or neighboring municipalities to harvest rice, corn, or plantain, or for social activities. ${ }^{8}$ In the last few years, people in Choco have been forced to move to other departments, but also to the town of Quibdo due to the worsening internal conflict. Malaria is reported throughout the year with peaks in the relatively drier months. Although vector densities are relatively low, Anopheles nuñeztovari is the vector species most frequently collected, followed by $A$. darlingi. ${ }^{9}$ They have been collected from rain puddles, lakes, and fishponds. Vector control activities are intermittent, and at the local level the malaria control program is based on treatment of all malaria cases. The use of bed nets is high $(68 \%$, mainly 
untreated), and there are frequent visits to health posts for malaria diagnosis $(85 \%$ of the people who had symptoms seek treatment in the public or private health sector). ${ }^{9}$ Nevertheless, self-medication is known to occur and both chloroquine and sulfadoxine-pyrimethamine are available over the counter in local pharmacies.

Study design and procedures. The study was a facilitybased case-control study. Cases were drawn from a malaria survey conducted in all four public and nine private health posts where malaria diagnosis was done in town, and stratified by health post to one control per case. Cases consisted of any person with a positive thick blood smear (regardless of parasite species), diagnosed between March 27 and July 27, 2001. Cases detected at the army and police clinics, a laboratory of an indigenous organization, and a temporary laboratory set up to take samples from displaced members of the population were considered to be so highly selected for the variables under study (e.g., high mobility or living outside town) that they were not included in the study.

Controls were randomly selected among individuals who visited a general practitioner in the same health facility where cases were detected, during the same period, but whose medical condition was not malaria. Individuals paying routine visits to the nurse or physician (e.g., for prenatal care, hypertension programs, etc.), or those who had had malaria or had taken antimalarial drugs in the previous month were excluded from the control group since these conditions were likely to affect their travel patterns and susceptibility to malaria. The number of controls in each health facility was matched to the number of cases on a weekly basis to adjust for seasonality of transmission.

Trained field workers interviewed cases and controls at the health facilities using a structured questionnaire. The questionnaire requested the patient's sociodemographic information, use of protective measures, malaria-related information, place of residence, and, if a resident of Quibdo, their history of traveling outside town within the preceding 12 months before disease onset.

Written informed consent was obtained from all participants. The project was reviewed and approved by the Ethical Committees of the International Center for Medical Research and Training in Colombia and the London School of Hygiene and Tropical Medicine in the United Kingdom.

Definition of traveling periods. The date of disease onset, the date of arrival, and the length of the stay in the places to which residents of the town of Quibdo had traveled before disease onset were used to derive a more detailed history of traveling in both cases and controls. For controls, there is, by definition, no date of malaria onset to which travel can be related. Moreover, since it is not an individually matched study, no particular case can be used to obtain such a date for each control. Therefore, a date was allocated by drawing a list of random numbers (STATA 7; Stata Corporation, College Station, TX) following a Poisson distribution, with mean equal to the duration of symptoms in the cases. This number was subtracted from the control's date of interview to generate a date of onset for each control.

The periods before disease onset in which individuals stayed outside Quibdo town were divided into intervals of one week during the first month (1-7, 8-14, 15-21, and 22-30 days), and then, in periods from 31 to 90,91 to 180 , and 181 to 365 days before disease onset. Each time interval was in- cluded as a single variable with the following code: $0=$ did not stay outside during this time interval, $1=$ stayed outside in a malaria-endemic area for at least one night during this time interval, and 2 = stayed outside in a non-endemic malaria area during this time interval. People staying outside Quibdo town in more than one period were included for all the corresponding intervals of time.

Data analysis. A descriptive analysis of cases and controls by health post was conducted, followed by univariate and multivariate analyses of risk factors for malaria. The odds ratios (ORs) and 95\% confidence intervals (CIs) for the risk factors for malaria were calculated by unconditional logistic regression models and stratified by health posts. For multivariate analyses, the models were fitted including all the explanatory variables and subsequently dropping them one by one. The final model was chosen with those variables that had a $P<0.1$ in the likelihood ratio test. However, occupation was not included in the final model because by definition it was associated with age (i.e., child was a category of occupation). For Plasmodium falciparum, an interaction between sex and health post was detected, and this interaction was included in the final logistic regression model.

Analyses were subdivided into $P$. falciparum and $P$. vivax because differences in the biology of Plasmodium species (such as the potential for relapses in $P$. vivax but not $P$. falciparum) meant that risk factors were expected to differ. Mixed $P$. falciparum and $P$. vivax infections were included in both species analyses.

The risk of malaria for those residents who stayed outside Quibdo town in an endemic area before disease onset was calculated based on a time interval of 8-14 days. This was chosen because it covers the typical incubation period for $P$. falciparum malaria (9-14 days). ${ }^{10}$ The adjusted population attributable risk of traveling to an endemic area in the 8-14 days before disease onset was calculated from each of the final logistic regression models for $P$. falciparum and $P$. vivax, according to Bruzzi and others. ${ }^{11}$ The relationship between malaria and traveling at time periods other than 8-14 days was explored by first restricting the analysis to cases and controls who had not stayed outside the town of Quibdo in an endemic area in the 8-14-day period before disease onset, and subsequently, to cases and controls who had not traveled to a malaria-endemic area at time intervals that showed a statistically significant association in the first analysis. The OR for time intervals of travel was each calculated in a separate model. The final logistic regression models used in the analysis of risk factors for $P$. falciparum and $P$. vivax were used. Each adjusted OR indicates the risk of malaria in those traveling to an endemic area at that time interval using the risk in those not traveling at that time interval as the baseline. Since individuals can have multiple travel episodes, these analyses were indirectly adjusted for frequency of traveling. The ratios in this study were compared using the Wald test. $\mathrm{P}$ values $<0.05$ were considered statistically significant.

\section{RESULTS}

During the study, 1,012 cases were diagnosed in the surveyed health facilities, from which $826(82 \%)$ were recruited. Seven hundred eighty controls were interviewed, but 10 were excluded because of a history of antimalarial drug use or a 
microscopically confirmed malaria episode in the previous month. Analyses of risk factors for malaria in 929 (395 cases and 534 controls) residents of Quibdo were carried out.

Analysis in residents of Quibdo town. In residents of Quibdo town, $285(72 \%)$ cases were due to $P$. falciparum alone, $94(24 \%)$ to $P$. vivax alone, and $16(4 \%)$ to mixed ( $P$. falciparum plus $P$. vivax) infections. The majority of cases $(72.8 \%)$ and controls $(97.2 \%)$ had not traveled to any place outside Quibdo town in the 8-14 days before disease onset. However, the frequency of traveling outside Quibdo to a malaria-endemic area in that period was much higher among cases $(26.4 \%)$ than controls $(1.7 \%)$. Six controls $(1.1 \%)$ and 3 cases $(0.8 \%)$ had traveled outside Quibdo but to an area non-endemic for malaria.

For $P$. falciparum, initial analyses showed that staying outside Quibdo town in a malaria-endemic area during the 8-14 days before disease onset was the strongest risk factor $(\mathrm{OR}=$ 26.6, 95\% CI = 13.15-53.73) (Table 1). In the adjusted model the association between $P$. falciparum malaria and staying outside Quibdo in an endemic area during the 8-14 days before disease onset remained very strong $(\mathrm{OR}=29,95 \%$ $\mathrm{CI}=14-60.32)$. Similarly, age (5-14 years old), male sex, a history of a malaria case at home in the previous month, and failure to use protection against mosquitoes continued to be positively associated with $P$. falciparum, while being $\geq 60$ years old remained a protective factor. In contrast, a history of a malaria episode in the previous year was no longer statistically significantly associated with $P$. falciparum malaria (Table 1).

For $P$. vivax, a history of malaria in the previous year (adjusted $\mathrm{OR}=4.2,95 \% \mathrm{CI}=2.3-7.8)$ and belonging to the Mestizo ethnic group (adjusted OR $=3.8,95 \% \mathrm{CI}=1.5-6.1$ ) were risk factors. Staying outside Quibdo during the 8-14 days before disease onset was also strongly associated with $P$. vivax (adjusted $\mathrm{OR}=14.2,95 \% \mathrm{CI}=5.3-38.5$ ), but not as strongly as with $P$. falciparum.

The adjusted population attributable fraction of traveling to an endemic area in the 8-14 days before disease onset for $P$. falciparum and for $P$. vivax among residents of Quibdo town was $30.2 \%(95 \% \mathrm{CI}=29-30.8 \%)$ and $11.8 \%(95 \%$ $\mathrm{CI}=10.3-12.4 \%)$, respectively.

Risk of malaria at different time periods. Among residents of Quibdo town, $45.8 \%$ (181 of 395) of the cases and $70 \%$ (374 of 534) of the controls had not stayed overnight outside the town in the year before disease onset, 34\% (134 of 395) of the cases and $20.6 \%$ (110 of 534) of the controls had stayed

TABLE 1

Univariate and multivariate analyses of risk factors for Plasmodium falciparum malaria in residents of Quibdo town, Colombia*

\begin{tabular}{|c|c|c|c|c|}
\hline Characteristic & $\begin{array}{c}\text { Cases } \\
\mathrm{n}=301(\%)\end{array}$ & $\begin{array}{c}\text { Controls } \\
\mathrm{n}=534(\%)\end{array}$ & $\begin{array}{c}\mathrm{OR}^{\dagger} \\
(95 \% \mathrm{CI})\end{array}$ & 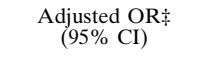 \\
\hline \multicolumn{5}{|l|}{ Age category (years) } \\
\hline$<1<-<1$ & $5(1.7)$ & $16(3)$ & $0.64(0.23-1.81)$ & $1.27(0.43-3.77)$ \\
\hline $1-4$ & $23(7.6)$ & $27(5.1)$ & $1.79(0.99-3.2)$ & $1.84(0.92-3.67)$ \\
\hline $5-14$ & $70(23.3)$ & $31(5.8)$ & $4.75(3-7.54)$ & $5.67(3.33-9.64)$ \\
\hline $15-44$ & $170(56.5)$ & $359(67.2)$ & 1 & 1 \\
\hline $45-59$ & $25(8.3)$ & $54(10.1)$ & $0.97(0.58-1.62)$ & $1.28(0.7-2.36)$ \\
\hline$\geq 60$ & $8(2.6)$ & $47(8.8)$ & $0.35(0.16-0.77)$ & $0.31(0.12-0.81)$ \\
\hline Sex & & $\mathrm{n}=533$ & & \\
\hline \multicolumn{5}{|l|}{ HSFA } \\
\hline Female & $24(8)$ & $41(7.7)$ & 1 & 1 \\
\hline Male & $22(7.3)$ & $42(7.9)$ & $0.89(0.43-1.84)$ & $0.59(0.24-1.46)$ \\
\hline \multicolumn{5}{|l|}{ Other health post } \\
\hline Female & $111(36.9)$ & $291(54.6)$ & 1 & 1 \\
\hline Male & $144(47.8)$ & $159(29.8)$ & $2.37(1.72-3.27)$ & $2.14(1.45-3.16)$ \\
\hline \multicolumn{5}{|l|}{ Race } \\
\hline Black & $264(87.7)$ & $472(88.7)$ & 1 & - \\
\hline White & $8(2.7)$ & $14(2.6)$ & $1.02(0.42-2.46)$ & - \\
\hline Indigenous & $6(2)$ & $4(0.7)$ & $2.68(0.75-9.6)$ & - \\
\hline Mestizo & $23(7.6)$ & $42(8)$ & $0.98(0.57-1.66)$ & - \\
\hline Occupation & & $\mathrm{n}=533$ & & \\
\hline Child & $34(11.3)$ & $42(7.9)$ & $2.33(1.34-4.06)$ & - \\
\hline Student & $98(32.6)$ & $143(26.8)$ & $2(1.3-3.02)$ & - \\
\hline Unemployed & $17(5.6)$ & $65(12.2)$ & $0.76(0.41-1.43)$ & - \\
\hline Housewife & $51(17)$ & $150(28.1)$ & 1 & - \\
\hline Outdoor worker & $30(4.3)$ & $26(2.8)$ & $3.41(1.84-6.3)$ & - \\
\hline Teacher & $4(1.3)$ & $15(2.8)$ & $0.78(0.25-2.46)$ & - \\
\hline Other & $67(22.3)$ & $92(17.3)$ & $2.16(1.38-3.38)$ & - \\
\hline Malaria last year & 49/297 (16.5) & $52(9.7)$ & $1.83(1.2-2.8)$ & $1.54(0.92-2.6)$ \\
\hline Case at home§ & $84 / 296(28.3)$ & $47 / 525(9)$ & $4.04(2.7-6.1)$ & $4.12(2.57-6.6)$ \\
\hline Holes in the walls or roof of the house & $249 / 298(83.5)$ & $403 / 532(75.7)$ & $1.63(1.13-2.36)$ & - \\
\hline Use of protection against mosquitoes & $198(65.7)$ & 408/533 (76.6) & $0.59(0.43-0.8)$ & $0.48(0.32-0.71)$ \\
\hline Stay outside town of Quibdo in the $8-14$ days before disease onset & $\mathrm{n}=300$ & $\mathrm{n}=530$ & & \\
\hline in a malaria endemic area & $94(31.3)$ & $9(1.7)$ & $26.6(13.15-53.73)$ & $29(14-60.32)$ \\
\hline in a non-endemic area & $2(0.7)$ & $6(1.1)$ & $0.84(0.17-4.22)$ & $0.99(0.2-5.14)$ \\
\hline did not stay outside & $204(68)$ & $515(97.2)$ & 1 & 1 \\
\hline
\end{tabular}

* CI = confidence interval; HSFA = Hospital San Francisco de Asís (main reference hospital)

$\dagger$ The odds ratio (OR) was stratified by health post, except for sex, where an interaction between sex and health post was detected.

$\ddagger$ The OR was adjusted for age, sex, use of protection, stay outside urban Quibdó in the 8-14 days prior to disease onset, history of malaria in the previous year, a malaria case at home in the previous month, health post, and the interaction between sex and health post.

$\S$ A history of a malaria case at home in the previous month. 
outside once, and the remaining $20.2 \%$ ( 80 of 395 ) of the cases and $9.4 \%$ (50 of 534) of the controls had stayed outside more than once (up to nine times).

When we restricted the analysis to the 204 cases and 515 controls who had not traveled in the 8-14 days before disease onset, Table 2 shows that for $P$. falciparum, individuals who traveled at 1-7, 15-21, or 22-30 days before disease onset had an increased risk of malaria. Table 2 also shows the adjusted OR for each other period of traveling before disease onset and $P$. falciparum malaria when the analysis was restricted to individuals who had not traveled in different periods. Traveling in the 1-7 days before disease onset was an important risk factor even when the analysis was restricted to those individuals who did not travel in the period 8-30 days before disease onset. A slightly increased risk of malaria was observed among those individuals who traveled in the period 22-30 days but had stayed in the town for the period 1-21 days before disease onset. However, this risk was no longer statistically significant, probably due to the small numbers of individuals who traveled. Approximately half of those away from Quibdo in the fourth week before illness were also away in the third week, which contributes to the small number of only fourth week travelers.
Based on these results, including all cases and controls, the risk of $P$. falciparum malaria was calculated for those individuals who traveled between 1 and 21 days before disease onset (adjusted OR $=24.15,95 \% \mathrm{CI}=14.1-41.2$ ) and those who traveled in the period 1-30 days before disease onset (adjusted OR $=21.32,95 \% \mathrm{CI}=12.8-5.5$ ).

For $P$. vivax, the risk of malaria was high in those individuals who traveled to an endemic area up to three months before disease onset. Traveling outside town in the period 91180 days before disease onset or earlier was not a risk factor.

Risk of malaria according to area of residence. The risks of being diagnosed with $P$. falciparum and $P$. vivax malaria among residents of areas outside Quibdo were compared with those of residents of the urban area who had not traveled outside town in the 8-14 days before disease onset. For $P$. falciparum, the analysis was adjusted by age, sex, race, having had a malaria case at home in the previous month, protection against mosquitoes, and health post. A history of a malaria episode in the previous year was added to the final logistic regression model for $P$. vivax.

The risk of $P$. falciparum in both residents of rural areas of Quibdo municipality and other municipalities of Choco was higher than that of residents of Quibdo who had not traveled

TABLE 2

Risk of Plasmodium falciparum malaria at different time intervals of traveling to a malaria-endemic area outside Quibdo town, Colombia before disease onset

\begin{tabular}{|c|c|c|c|c|}
\hline \multirow[b]{2}{*}{ Group of analysis* } & \multicolumn{2}{|c|}{ Number (\%) of exposed } & \multirow[b]{2}{*}{$\begin{array}{l}\text { Periods of traveling (days) } \\
\text { before disease onset }\end{array}$} & \multirow[b]{2}{*}{$\begin{array}{c}\text { Adjusted OR }{ }^{(95 \% \mathrm{CI})} \\
\end{array}$} \\
\hline & $\begin{array}{c}\text { Cases } \\
\mathrm{n}=204\end{array}$ & $\begin{array}{l}\text { Controls } \\
\mathrm{n}=515\end{array}$ & & \\
\hline \multirow[t]{7}{*}{ Did not travel 8-14 days } & $26(12.7)$ & $9(1.7)$ & $1-7$ & $11.93(5.12-27.8)$ \\
\hline & $11 / 203(5.4)$ & $4 / 509(0.8)$ & $15-21$ & $11.81(3.33-41.91)$ \\
\hline & $11 / 203(5.4)$ & $6 / 510(1.2)$ & $22-30$ & $6.5(2.14-19.73)$ \\
\hline & $21 / 203(10.3)$ & $29 / 506(5.7)$ & $31-90$ & $1.69(0.87-3.26)$ \\
\hline & $19 / 203(9.4)$ & $29 / 505(5.7)$ & $91-180$ & $1.78(0.9-3.53)$ \\
\hline & $17 / 203(8.4)$ & 29/504 (5.7) & $181-365$ & $1.37(0.67-2.8)$ \\
\hline & Cases $\mathrm{n}=193$ & Controls $\mathrm{n}=511$ & & \\
\hline \multirow[t]{6}{*}{ Did not travel 8-21 days } & $25(13)$ & $9(1.8)$ & $1-7$ & $13.51(5.74-31.8)$ \\
\hline & $4 / 192(2.1)$ & $4 / 506(0.8)$ & $22-30$ & $2.52(0.55-11.48)$ \\
\hline & $14 / 192(7.3)$ & $28 / 502(5.6)$ & $31-90$ & $1.04(0.49-2.22)$ \\
\hline & 13/192 (6.8) & 28/501 (5.6) & $91-180$ & $1.17(0.54-2.53)$ \\
\hline & $16 / 192(8.3)$ & $28 / 500(5.6)$ & $181-365$ & $1.4(0.67-3)$ \\
\hline & Cases $\mathrm{n}=189$ & Controls $\mathrm{n}=507$ & & \\
\hline \multirow[t]{5}{*}{ Did not travel 8-30 days } & $24(12.7)$ & $9(1.8)$ & $1-7$ & $13.38(5.66-31.64)$ \\
\hline & $10 / 188(5.3)$ & $25 / 498(5)$ & $31-90$ & $0.8(0.34-1.9)$ \\
\hline & $13 / 188(7)$ & $26 / 497(5.2)$ & $91-180$ & $1.3(0.59-2.84)$ \\
\hline & $15 / 188(8)$ & $27 / 496(5.4)$ & $181-365$ & $1.38(0.64-2.97)$ \\
\hline & Cases $\mathrm{n}=215$ & Controls $\mathrm{n}=511$ & & \\
\hline \multirow[t]{7}{*}{ Did not travel 1-7 days } & $38(17.7)$ & $3(0.6)$ & $8-14$ & $51.8(15.14-177.48)$ \\
\hline & $30 / 214(14)$ & $7 / 505(1.4)$ & $15-21$ & $16.4(6.6-40.76)$ \\
\hline & $17 / 214(8)$ & $7 / 506(1.4)$ & $22-30$ & $7.13(2.65-19.2)$ \\
\hline & $22 / 213(10.3)$ & $29 / 502(5.8)$ & $31-90$ & $2.04(1.07-3.87)$ \\
\hline & $19 / 213(9)$ & 28/501 (5.6) & $91-180$ & $1.75(0.88-3.48)$ \\
\hline & 13/213 (6.1) & $27 / 500(5.4)$ & $181-365$ & $0.92(0.41-2.04)$ \\
\hline & Cases $\mathrm{n}=178$ & Controls $\mathrm{n}=500$ & & \\
\hline \multirow[t]{6}{*}{ Did not travel 1-14 days } & $10(5.6)$ & $4(0.8)$ & $15-21$ & $15(4.13-54.35)$ \\
\hline & $9 / 178(5)$ & $6 / 501(1.2)$ & $22-30$ & $7.15(2.26-22.62)$ \\
\hline & $14 / 177(8)$ & $28 / 497(5.6)$ & $31-90$ & $1.5(0.72-3.11)$ \\
\hline & $14 / 177(8)$ & $28 / 496(5.6)$ & $91-180$ & $1.5(0.71-3.24)$ \\
\hline & $12 / 177(6.8)$ & $27 / 495(5.4)$ & $181-365$ & $1.06(0.47-2.43)$ \\
\hline & Cases $\mathrm{n}=168$ & Controls $\mathrm{n}=497$ & & \\
\hline \multirow[t]{4}{*}{ Did not travel 1-21 days } & $3(1.8)$ & $4(0.8)$ & $22-30$ & $2.16(0.4-11.6)$ \\
\hline & $8 / 167(4.8)$ & $27 / 493(5.5)$ & $31-90$ & $0.75(0.31-1.84)$ \\
\hline & $9 / 167(5.4)$ & $27 / 492(5.5)$ & $91-180$ & $0.83(0.34-2.04)$ \\
\hline & $11 / 167(6.6)$ & $26 / 491(5.3)$ & $181-365$ & $1.03(0.43-2.47)$ \\
\hline
\end{tabular}

Analysis was restricted to the group described.

$\dagger$ The odds ratio (OR) was adjusted for age, sex, use of protection, history of malaria in the previous year, a malaria case at home in the previous month, health post, and the interaction between sex and health post. $\mathrm{CI}=$ confidence interval. 
to a malaria-endemic area. With respect to $P$. vivax, inhabitants of the rural areas of Quibdo did not show an increased risk, but residents of other municipalities did show an increased risk (Table 3 ).

\section{DISCUSSION}

In this study, traveling outside town was identified as the strongest risk factor for malaria in the town of Quibdo. Temporary movements of residents of areas where malaria transmission exists to other endemic areas (usually with comparatively higher transmission intensity) has been associated with an increase in the risk of malaria in different scenarios. For example, a history of traveling was a risk factor for $P$. falciparum malaria in a refugee camp in Thailand, ${ }^{12}$ and urban areas in Africa, ${ }^{3,4}$ and for both $P$. falciparum and $P$. vivax infections in rural settlements in Brazil and Colombia. ${ }^{13,14}$ Our results were comparable to those of Luo in 2000 in the highlands of China, where temporary migration was also the most important risk factor for both $P$. falciparum (adjusted rate ratio $=15.7,95 \% \mathrm{CI}=8.7-28.3$ ) and $P$. vivax (adjusted rate ratio $=4.9,95 \% \mathrm{CI}=3.5-6.9$ ) infections (Luo DP, unpublished data). Temporary migration in the cohort study of Luo was defined as staying outside the village of residence for at least one night, in an area considered of higher risk of malaria, during the seven months of the study. The definition of temporary migration in the study of Luo and the present study differ only in that we restricted our initial analysis to the 8-14 days period prior to disease onset. This interval was based on the typical incubation period of both $P$. falciparum and $P$. vivax, since the objective of our study was to identify those residents who were more likely to have acquired malaria outside town (inferred imported cases). However, when residents who had traveled to endemic areas in the 8-14-day period were excluded, the risk of $P$. falciparum malaria was also high among those who had traveled up to one month previously, and up to three months for $P$. vivax. The higher risk of infection in periods longer than 14 days could be explained by the longer incubation periods observed in both species, ${ }^{10}$ and some $P$. vivax cases being relapses. We do not know of any reports of very short incubation periods $(<7$ days) resulting from sporozoite infections. Thus, the higher risk of disease among those who traveled outside town 1-7 days prior to disease onset, but not 8-30 days before, is probably the result of patients reporting only the most recent trip

TABLE 3

Risk of malaria (by parasite species) in residents of areas outside of the town of Quibdo, Colombia in comparison with residents of Quibdo who did not travel to a malaria-endemic area in the 8-14 days before disease onset

\begin{tabular}{lcc}
\hline \multicolumn{1}{c}{ Area of residence } & $\begin{array}{c}\text { Plasmodium } \\
\text { falciparum } \\
\text { Adjusted OR } \\
(95 \% \mathrm{CI}) *\end{array}$ & $\begin{array}{c}P . \text { vivax } \\
\text { Adjusted OR } \\
(95 \% \mathrm{CI}) \dagger\end{array}$ \\
\hline Urban area and did not travel & 1 & 1 \\
Rural area of Quibdo & $2.65(1.86-3.77)$ & $0.7(0.31-1.24)$ \\
Other municipality & $4.35(3.11-6.08)$ & $3.41(2.05-5.67)$ \\
Other department & $2.5(0.84-7.48)$ & $1.23(0.2-7.37)$ \\
\hline
\end{tabular}

* The odds ratio (OR) adjusted for age, sex, race, use of protection against mosquitoes, having had a malaria case at home in the previus month, and health post.

$\dagger$ The OR adjusted for age, sex, race, use of protection against mosquitoes, having had a malaria case at home in the previous month, a history of a malaria episode in the previous year, and health post. but not previous ones (when they might have been exposed), or inaccurate disease onset or traveling dates. The nature of the case-control study did not allow us to validate the dates reported by the patients, for which a cohort study would be required. In the meantime, self-defined traveling to an endemic area 1-21 days or 1-30 days prior to disease onset rather than the dates covering the typical incubation period for malaria (8-14 days) seems to be a good indicator of malaria risk among local residents and could be a suitable definition of imported cases in residents of Quibdo. Asking patients whether they have been in a malaria-endemic area outside town in the month before disease onset would be relatively easy to apply when defining imported malaria in the field.

With respect to malaria control, it would be expected that targeting prevention measures to residents who travel to endemic areas outside town could considerably reduce malaria incidence (mainly $P$. falciparum) among inhabitants of Quibdo. Use of protection measures (mainly bed nets) reduced the risk of $P$. falciparum in residents, which suggests that this is a worthwhile control measure for the study area. The ownership of bed nets (mainly untreated with insecticide) is relatively high in Quibdo town (more than 60\%), so the local authorities may consider strategies to improve their use and coverage, and to encourage insecticide impregnation. The lack of a protective effect of bed nets in $P$. vivax malaria is a common finding, usually attributable to the presence of relapses. $^{15}$

The history of a malaria case at home as a risk factor for malaria could indicate susceptibility among inhabitants of the same household. This susceptibility might be genetic, behavioral, or sociodemographically determined. ${ }^{16}$ The highest risk of malaria being in children 5-14 years old, together with the protective effect of being $\geq 60$ years old, suggests that residents of Quibdo town are able to eventually develop some degree of acquired immunity, in spite of the low intensity of transmission.

The relationship between ethnic group and $P$. vivax, but not $P$. falciparum, is most likely explained by the rarity of Duffy antigen among the black community in Quibdo town. Similarly, the ability of $P$. vivax to relapse is the most likely reason for the association between history of malaria in the previous year and risk of infection with this species.

Residents of areas outside the town had a higher risk of being diagnosed with $P$. falciparum malaria in the health facilities of Quibdo than local residents who did not travel 8-14 days before disease onset. For $P$. vivax, this risk was higher only for residents of other municipalities of Choco. The variations in the risk of malaria with place of residence are probably related to the risk of infection by each species in the different municipalities in Choco. Similar results were reported by Lansang and others ${ }^{17}$ in a low-intensity transmission area in The Philippines, and by Fungladda and others ${ }^{18}$ in Thailand. In the former study, the risk of a positive blood slide was strongly correlated with the level of transmission (measured by seroprevalence) in the area of residence (adjusted $\mathrm{OR}=5.18,95 \% \mathrm{CI}=3.49-7.68)$. Likewise, Fungladda and others found that individuals residing in forested areas were at a higher risk of malaria than those individuals who did not have contact with the forest (adjusted $\mathrm{OR}=10.25,95 \% \mathrm{CI}=6.28-16.74)$. In spite of a similar study design, the ORs found in our study were lower than those 
reported in Thailand, but the detailed epidemiology of the areas differs and residents of Quibdo are also exposed to some local malaria transmission that could partly explain this difference. Direct measurements of the risk of malaria in areas outside Quibdo would have been ideal, but it could not be conducted due to security reasons.

Since it appears that in Quibdo most $(>80 \%)$ people who experience malaria symptoms seek treatment in a public or private clinic, ${ }^{9}$ the selection of cases in health posts is likely to include most cases detected in the area. However, a proportion of cases inevitably remain undetected. Active search for febrile individuals in the community could have been conducted to detect these cases, but the large size of the area (population $>90,000$ ) and the relatively low incidence of malaria made active case detection impracticable. The sensitivity in the detection of cases depends on the method of diagnosis, which in the case of malaria (thick blood smear) relies very much on the expertise of the technician. All the health workers who examined the thick blood smears had several years of experience and were under constant supervision by the local reference laboratory. Quality control of malaria diagnosis is routinely performed in most municipalities in Colombia (including Quibdo), and a recent study shows that the overall performance of diagnosis in the country is of a high standard (kappa index $=0.84) .{ }^{19}$ Nevertheless, errors cannot be avoided completely, either in the diagnosis of cases or in the classification of parasite species.

For studying population movement, a clear definition of the spatial and temporal boundaries is required. In this study, the spatial scale was set as the urban area of Quibdo (defined in a map of the area), and the temporal scale as all movements of at least one day in the year prior to disease onset. Shorter movements during daylight (especially those at dawn when the mosquito is active), or to the forest surrounding the urban area, may also carry a risk but were not included in the study. Exposure was defined as traveling outside Quibdo to or living in, an endemic area. The endemic and non-endemic areas were classified based on the reports to the malaria control program, but two factors could affect the sensitivity of this definition. First, the intensity of malaria transmission may vary over time, and thus the risk of infection. Second, malaria transmission is highly focal ${ }^{16}$ and patients may have visited a village where there was no transmission, although the municipality to which that village belonged had been classified as endemic based on the transmission in other villages. Nevertheless, the conditions for malaria transmission exist in most of the department of Choco and the risk of malaria is believed to be higher than in Quibdo.

As previously mentioned, validation of movement is not feasible in case-control studies. Information bias can occur if cases or controls do not want to report their movements (for example those related to illegal activities). We did not notice this as a major problem but it cannot be ruled out. Patients were asked about their traveling patterns after they had received the diagnosis. The cases could have been motivated by the diagnosis to recall their movements. However, the control group was also under similar circumstances when they were interviewed (i.e., attending a clinic), and this minimizes the chances of recall bias.

In conclusion, traveling outside the urban area was the strongest risk factor for malaria among inhabitants of the town of Quibdo. The results are useful for the development of a definition of imported and autochthonous malaria. With this definition, malaria cases diagnosed in Quibdo can be usefully classified into autochthonous and imported, thereby allowing the urban malaria to be monitored and methods of prevention and control to be assessed.

Received November 21, 2003. Accepted for publication April 22, 2004.

Acknowledgments: We acknowledge the help and cooperation of the health authorities of Choco department and Quibdo municipality, and the personnel of the public and private health facilities during the conduction of the study. We are grateful to Dr. Iveth Gonzalez, Dr. Maria Helena Chacon, Rubiela Giraldo, the field workers, and the individuals who participated in the study.

Financial support: This study was supported by the United Nations Children's Fund/United Nations Development Program/World Bank/ World Health Organization Special Program for Research and Training in Tropical Diseases contract no. M8/181/4/O184. Lyda Osorio received a training grant from the Instituto Colombiano para el Desarrollo de la Ciencia y la Tecnologia Francisco Jose de Caldas, and David J. Bradley is a member of the United Kingdom Department for International Development Research Program on Malaria Control.

Authors' addresses: Lyda Osorio, International Center for Medical Research and Training, Avenida 1, Norte \#3-03, Cali, Colombia, Telephone: 57-2-668-2164, Fax: 57-2-667-2989, E-mail: lyda.osorio@ lshtm,ac.uk. Jim Todd and David J. Bradley, Department of Infectious and Tropical Diseases, London School of Hygiene and Tropical Medicine, Keppel Street, London WC1E 7HT, United Kingdom, Telephone: 44-20-7612-7870, Fax: 44-20-7636-8739, E-mail: jim. todd@1shtm.ac.uk, Telephone: 44-20-7927-2233, Fax: 44-20-75809075, E-mail: david.bradley@1shtm.ac.uk.

Reprints requests: Lyda Osorio, International Center for Medical Research and Training, Avenida 1, Norte \#3-03, Cali, Colombia

\section{REFERENCES}

1. Prothero R, 1965. Migrants and Malaria. London: Longmans.

2. Robert V, Macintyre K, Keating J, Trape J, Duchemin JB, Warren M, Beiber J, 2003. Malaria transmission in urban SubSaharan Africa. Am J Trop Med Hyg 68: 169-176.

3. Watts T, Wray J, Ng'andu N, Draper C, 1990. Malaria in an urban and a rural area of Zambia. Trans $R$ Soc Trop Med Hyg 84: 196-200.

4. Jambou R, Tombo ML, Raharimalala L, Rakotonjanabelo A, Rabe T, Laventure S, Boisier P, 1998. Malaria in Antananarivo: evaluation of a post-epidemic situation. Sante 8: 257264.

5. Mendez F, Carrasquilla G, Muñoz A, 2000. Risk factors associated with malaria infection in an urban setting. Trans $R$ Soc Trop Med Hyg 94: 367-371.

6. Carme B, 1993. Plasmodium falciparum malaria in urban zones of high endemic regions in black Africa. Potential seriousness and possible preventive measures. Bull Soc Pathol Exot 86 : 394-398.

7. Montoya R, 1999. Comportamiento de la malaria durante 1999. Informe Quincenal Epidemiológico Nacional. Santafe de Bogota DC: Ministerio de Salud de Colombia, Instituto Nacional de Salud, 274-279.

8. Jimeno M, Sotomayor M, Valderrama L, 1995. Choco: Biodiversidad Cultural y Medio Ambiente. Santafe de Bogota DC. Bogota: Fondo Financiera Electrica Nacional.

9. Serra M, Gómez E, Carvajal R, Banguero M, Olano V, Córdoba F, 1999. Epidemiología de Malaria en el Área Urbana y Rural de Quibdo e Istmina. Quibdo: Fundación para la Educaci. Superior, División Salud.

10. Harinasuta T, Bunnang D, 1988. The clinical features of malaria. Wernsdorfer WH, McGregor SI, eds. Malaria. Principles and Practice of Malariology. London: Churchill Livingstone, 709779.

11. Bruzzi P, Green SB, Byar DP, Brinton LA, Schairer C, 1985. 
Estimating the population attributable risk for multiple risk factors using case-control data. Am J Epidemiol 122: 904-914.

12. Somboon P, Aramrattana A, Lines J, Webber R, 1998. Entomological and epidemiological investigations of malaria transmission in relation to population movements in forest areas of north-west Thailand. Southeast Asian J Trop Med Public Health 29: 3-9.

13. Camargo LM, Ferreira MU, Krieger H, de Camargo EP, da Silva LP, 1994. Unstable hypoendemic malaria in Rondonia (western Amazon region, Brazil): epidemic outbreaks and workassociated incidence in an agro-industrial rural settlement. $\mathrm{Am}$ J Trop Med Hyg 51: 16-25.

14. Sevilla-Casas E, 1993. Human mobility and malaria risk in the Naya River basin of Colombia. Soc Sci Med 37: 1155-1167.

15. Rowland M, Webster J, Saleh P, Chandramohan D, Freeman T, Pearcy B, Durrani N, Rab A, Mohammed N, 2002. Prevention of malaria in Afghanistan through social marketing of insecti- cide-treated nets: evaluation of coverage and effectiveness by cross-sectional surveys and passive surveillance. Trop Med Int Health 7: 813-822.

16. Greenwood B, 1989. Impact of culture and environmental changes on epidemiology and control of malaria and babesiosis. The microepidemiology of malaria and its importance to malaria control. Trans $R$ Soc Trop Med Hyg 83: 25-29.

17. Lansang M, Belizario V, Bustos M, Saul A, Aguirre A, 1997. Risk factors for infection with malaria in a low endemic community in Bataan, the Philippines. Acta Trop 63: 257-265.

18. Fungladda W, Sornmani S, Klongkamnuankarn K, Hungsapruek T, 1987. Sociodemographic and behavioural factors associated with hospital malaria patients in Kanchanaburi, Thailand. $J$ Trop Med Hyg 90: 233-237.

19. Garcia M, Mendoza N, 2002. Evaluation of the Malaria Evaluation Program in the national laboratory network in Colombia. Biomedica (Bogota) 22: 123-132. 